\title{
Routing metrics for Wireless Mesh Networks
}

\author{
Georgios Parissidis, Merkourios Karaliopoulos, Rainer Baumann, \\ Thrasyvoulos Spyropoulos
}

\author{
Computer Engineering and Networks Laboratory \\ ETH Zurich, Switzerland
}

\begin{abstract}
Routing in wireless mesh networks has been an active area of research for many years. Among the routing protocols proposed, a large majority selects paths that minimize hop count. Whereas minimum hop count is the most popular metric in wired networks, in wireless networks interference- and energy-related considerations give rise to more complex trade-offs. Therefore, a variety of routing metrics has been proposed for wireless mesh networks providing routing algorithms with high flexibility in the selection of best path and offering a compromise between throughput, end-to-end delay, and energy consumption. In this paper, we present a detailed survey and taxonomy of routing metrics. These metrics can have broadly different optimization objectives (e.g. to optimize application performance, maximize battery lifetime, maximize network throughput), different methods to collect the required information to produce metric values, and different ways to derive the end-to-end route quality out of the individual link quality metrics. The presentation of the metrics is highly comparative, with emphasis on the strengths and the weaknesses of both individual and whole families of metrics. We also discuss the main implications for practitioners and identify open issues for further research in the area.
\end{abstract}




\section{Introduction}

Routing in wireless mesh networks has been a highly popular research topic during the last decade. Whereas many routing function objectives are the same as in the of wired networks and the Internet, wireless mesh networks add several new dimensions that make the problem less straightforward and more interesting at the same time. As a result, although experience and wisdom gained by wired networks have guided the first steps in the wireless domain, in many cases there was need for novel approaches and solutions.

In the Internet, routing protocols are quite stable; changes in connectivity may happen but are not frequent. As a result, routing protocols for wired networks proactively maintain routes from all nodes to every other node, by propagating the occasional topology update as soon as it occurs. However, the topology of wireless mesh networks changes much more dynamically than in wired networks. This is primarily due to node mobility on the one hand, e.g. in mobile ad hoc networks or hybrid networks with both mobile and static nodes, and the impairments of wireless links due to propagation phenomena, on the other hand. These two phenomena result in wireless networks being often only intermittently connected, which makes the use of proactive routing protocols and the overhead related to route maintenance less attractive.

We summarize here the additional challenges related to wireless mesh networks:

Node mobility. Wireless mesh nodes may move. As a result, links may break and network topology may change frequently; in graph-theoretic terms, the connectivity graph varies more quickly with time. This makes route maintenance much more complex than in wired networks.

Wireless propagation phenomena. In the wireless environment, node transmissions are physically broadcast and subject to radio propagation dynamics, such as shadowing, fading, etc. This makes even static link quality fluctuate over time, and can also result in the emergence of grey zones in the network, where links have bad quality but may still allow some successful transmissions. Situations like these trigger frequent route re-establishments.

Energy constraints. In many cases, energy preservation and elongation of battery lifetime may become the primary objectives for network operation. Advances in battery technology are significantly slower than those in nanotechnology and electronics. Thus, the available power will continue to be a performance bottleneck for handheld, low-end devices and sensors, in scenarios where nodes move and operate for long periods without access to the electricity grid.

Lack of centralized control. One of the most attractive features of wireless mesh networks is self-organization. Various functions, such as medium access 
control and routing, are carried out in a fully distributed manner with minimal human intervention. They are not subject to any centralized network management processes of the kind practiced in wired networks. However, the drawback is that most decisions are made by individual nodes having primarily knowledge about their local environment only. This leaves little margin for network optimizations that require global knowledge about the network state. More critically, the network operation itself assumes the cooperation of all nodes, rendering the network more vulnerable to node misbehavior practices.

The need to think differently when it comes to wireless mesh networks is also reflected in the large variety of routing metrics that have been proposed along with routing protocols, in order to enable efficient data delivery in the wireless context. This does not mean that routing metrics in wired networks are not in abundance. Besides minimum hop count (shortest path first), which is the alma matter of metrics, the literature is quite rich in other metrics that have either been more "intelligent" in pursuing minimum delivery delay or have prioritized other aspects of network performance [1]. Load sharing and balancing, fault-tolerance, low jitter, and high throughput rank high on the list of goals that have determined the costs of links and paths in the network and have driven the routing decisions. Whereas these objectives remain relevant in wireless mesh networks, there are additional concerns that may complement or overshadow traditional objectives.

This chapter identifies different categories of routing metrics proposed for wireless mesh networks and describes the rationale of each category. Some metrics are treated in more detail, either because they were the first to introduce a new approach or because they are being considered, themselves or their variations, in standardization procedures. Our description is deliberately comparative, pointing to the similarities and differences amongst the different categories and the relative advantages of each metric. Wherever appropriate, we draw references to studies that have already made such comparisons between the metrics discussed.

\section{Background}

It is possible to group routing metrics into broader categories according to a number of criteria. The optimization goal, the way required information for the metric computation is collected, and the way the route (path) metric is related to individual link metrics, have been selected as a non-exhaustive list of attributes for organizing the presentation of metrics. 


\subsection{Optimization objectives}

A routing metric is essentially a value assigned to each route or path, and used by the routing algorithm to select one, or more, out of a subset of routes discovered by the routing protocol. These values generally reflect the cost of using a particular route with respect to some optimization objective, and could take into account both application and network performance indicators. More specifically, the objective of the routing algorithm and thus the routing metric may be to:

- Minimize delay. This is often the canonical objective of the routing function. The network path over which the data can be delivered with minimum delay is selected. If queuing delays, link capacity, and interference are not taken into account, then delay minimization often ends up being equivalent to hop-count minimization.

- Maximize probability of data delivery. For non real-time applications, the main requirement is to achieve a low data loss rate along the network route, even at the expense of increased delay. This is equivalent to minimizing the probability of data loss between network end-points.

- Maximize path throughput. Here, the aim is the selection of an end-to-end path that consists of links with high capacity.

- Maximize network throughput. Contrary to the first three objectives, which are user application-oriented, network throughput is a system objective. The objective may be formulated as the maximization of data flow in the whole network or, implicitly, through the minimization of interference or retransmissions.

- Minimize energy consumption. Energy consumption is rarely an issue in wired networks. However, it becomes a major concern in sensor networks and mobile ad hoc networks, where the battery lifetime constrains the autonomy of network nodes.

- Equally distribute traffic load. This objective is more general. Here, the aim is to ensure that no node or link is disproportionately used, and could be achieved, for example, by minimizing the difference between the maximum and minimum traffic load over the network links. Load balancing may have an indirect effect on other objectives such as battery lifetime, per node throughput, etc.

It is worthwhile to note here that the first three objectives in the above list are concerned with individual application performance, that is, to optimize the performance for a given end-to-end path, while the last three are "system-oriented" objectives that focus on the performance of the network as a whole. Furthermore, routing metrics may consider more than one of the aforementioned objectives. In this case, the multi-dimensional metric combines the different measures, weighting them appropriately to account for the relative prioritization of the objectives. 


\subsection{Link and Path metrics}

The ultimate decision to be made by routing will be about the selected route(s); therefore, the final metric value that will be the subject of comparison will relate to the whole route (path). However, the path metric needs to be somehow derived as a function of the individual metric values estimated for each link in the path. The actual function to be used varies and highly depends on the actual metric in question. The most widely used functions are:

- Summation. The link metric values are added to yield the path metric. Examples of additive metrics are the delay or number of retransmissions experienced over a link.

- Multiplication. Values estimated over individual links are multiplied to get the overall path metric. The probability of successful delivery is an example of a multiplicative metric.

- Statistical measures (minimum, maximum, average). The path metric coincides with the minimum, average, or the maximum of values encountered over the path links. Example of the first case is the path throughput, which is dictated by the minimum link throughput (bottleneck link) over all hops included in a network path.

\subsection{Metric computation method}

There are also various ways in which network nodes acquire the information they need for the computation of the routing metric:

- Reuse of locally available information. Information required by the metric is available locally at the node, usually as result of the routing protocol operation. Such information may include the number of node interfaces, number of neighbor nodes (degree), length of input and output queues.

- Passive Monitoring. Information for the metric is gathered by observing the traffic coming in and going out of a node. No active measurements are required. In combination with other measurements, this can be used, for example, to estimate the available bandwidth.

- Active probing. Special packets are generated, whose function is to measure the properties of a link/path. This method incurs the highest overhead on the network, which is directly dependent on the frequency of measurements.

- Piggyback probing. This method also involves measurements. However, these measurements are now carried out by including probing information into regular traffic or routing protocol packets. With piggyback probing, no additional packets are generated for metric computation purposes, thus reducing the over- 
head for the network. Piggyback probing is a common method to measure delay.

Raw information about a link, acquired from passive or active measurements, usually requires some processing before it can be used to construct efficient and stable link metrics. Measured network parameters (e.g., delay or link loss ratio) are often subject to high variation. It is usually desired that short-term variations do not influence the value of a metric; otherwise, rapid oscillations of the metric value could, depending on the actual metric context, may result in the phenomenon of self-interference, quite early observed in Internet applications [2]: once a link is recognized as good, it is chosen by the routing protocol and starts getting used till is overloaded and is assigned with a worse metric value. As traffic starts to route around this link, its metric value increases again and the effects starts anew.

Therefore, metric measurements are subject to some filtering over time. Different metrics apply different types of filtering:

- Dynamic History Window: an average is computed over a number of previous measurement samples, which varies depending on the current transmission rate.

- Fixed History Interval: an average is computed over a fixed number of previous measurement samples.

- Exponential Weighting Moving Average (EWMA): measurement samples are weighted so that the impact of past samples on the current value of the metric decays exponentially with the sample age. Every time a new sample $d$ is obtained, the value of the metric is updated as: $d_{\text {new }}=\alpha \cdot d_{\text {old }}+(1-\alpha) \cdot d_{\text {sample }}$ with $\alpha \in[0,1]$ being the weighting factor, and $d_{\text {old }}$ the current metric value.

\section{Routing Metrics}

In this section we describe routing metrics proposed for wireless mesh networks. Firstly, we discuss topology-based metrics and demonstrate the performance disadvantage of the hop count metric in wireless mesh networks. We then argue in favor of more elaborate metrics that can address the additional challenges of wireless mesh networks and present the main metrics proposed up-to-date in literature. The presentation groups metrics in four categories, namely i) receiving signal strength based, ii) use of active probing measurements, iii) mobility-awareness, and iv) energy-awareness. 


\subsection{Topology based}

The main advantage of topology-based routing metrics is their simplicity. Examples of relevant topological information are the number of neighbors of each node, and the number of hops and/or paths towards a particular destination. The metrics almost always take into account connectivity information that becomes available locally by the routing protocol, without requiring additional passive or active measurements.

In general, the topology definition in wireless networks is less straightforward than in wired networks. First of all, links are physically broadcast. The link definition between two nodes is a soft definition; a link is said to exist as long as the one node is within the transmission range of the other, which is a function of the sender node transmit power, the reception sensitivity of the receiving node and the propagation environment. In fact, varying the transmit power of nodes lies at the heart of the topology control function, an important tool for engineering wireless mesh networks.

Another complication in wireless mesh networks is related to the link asymmetry. Although node $\mathrm{X}$ may receive successfully packets from node $\mathrm{Y}$, it may well be that node $\mathrm{Y}$ cannot receive packets of node $\mathrm{X}$. The reason is different interference levels at the neighborhood of the two nodes. This asymmetry has to be taken into account when making routing decisions, in particular for bidirectional traffic (e.g., TCP traffic).

Although topology-based metrics do not take into account several variables that have an impact on both the network and application performance, such as the transmit rates of the links are popular due to their simplicity. In fact, one of them, the hop-count metric, is by far the most popular metrics in wired networks and one of the first to consider in wireless mesh networks as well.

\subsubsection{Hop count}

The concept of the hop count metric is simple: every link counts as one equal unit, independent of the quality or other characteristics of the link. The ease of implementation has made hop count the most widely used metric in wired networks; it is implicitly or explicitly the default metric in many popular wireless mesh network routing protocols, such as OLSR [3], DSR [4], DSDV [5] and AODV [6].

The rationale for minimizing the hop metric is straightforward. Fewer hops on the data path imply smaller delay (higher throughput) and reduced waste of network resources, whether these involve network links or buffers or computational power. The implicit assumption is the existence of error-free links, which is almost always the case with wired networks.

On the contrary, links in wireless mesh networks cannot be assumed error-free. The wireless radio propagation environment, external and network-internal inter- 
ference, and, when relevant, the node mobility result in intermittent connectivity between the network nodes. Minimum hop count tends to select more distant nodes. Depending on the flexibility in setting the transmit power, a node has two options:

- The node may increase the transmit power to achieve a target probability of successful delivery despite the large distance to the receiver. The result of the minimum-hop count in this case is increased power consumption, which may be a concern for low-end, battery-powered devices.

- On the other hand, when the transmit power is fixed, the probability of data loss over the more distant link increases (on average). The risk of retransmissions is higher, implying additional energy consumption at the node, more interference at the network, and, eventually, increased delay. We illustrate this scenario with a simple example below.

\section{Example 1:}

a) Assume that the probability of packet loss between the node S-D in Fig.1 is $p_{1}$ in both directions, $S \rightarrow D$ and $D \rightarrow S$. Likewise, the probability of loss over both hops of path SHD is $p_{2}$, again in both directions. A packet transmission is considered successful when the data packet is correctly received in the forward direction and an ACK packet is correctly received in the reverse direction, as in the unicast $802.11 x$ transmission mode. What would be the minimum value of loss $p_{1}$, under which the minimum-hop path SD would result in larger delay than the two-hop path SHD? Assume, for simplicity, that the number of retransmissions at the linklayer is infinite.

\section{Answer:}

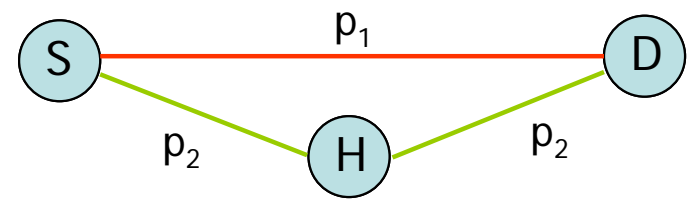

Fig. 1. One-hop path vs. two-hop path

Given that the propagation delay is small (in the order of sec) compared to the transmission delay (in the order of msec), the overall end-to-end delay of the packet is directly proportional to the total number of hop transmissions (including retransmissions) along the path.

The number of transmissions over a hop with symmetric packet probability loss, i.e., $p_{f}=p_{r}=p$, is a Geometric RV with parameter $(1-p)^{2}$; the mean ex- 
pected number of transmissions, assuming infinite retransmissions, equals $1 /(1-p)^{2}$.

The end-to-end normalized delay over paths SD and SHD are:

$$
D_{S D}=\frac{1}{\left(1-p_{1}\right)^{2}} \text { and } D_{S H D}=\frac{2}{\left(1-p_{2}\right)^{2}}
$$

Therefore,

$$
D_{S D} \geq D_{S H D} \Rightarrow \frac{1}{\left(1-p_{1}\right)^{2}} \geq \frac{2}{\left(1-p_{2}\right)^{2}} \Rightarrow \frac{\left(1-p_{2}\right)}{\left(1-p_{1}\right)} \geq \sqrt{2} \Rightarrow p_{1} \geq \frac{\sqrt{2}-1+p_{2}}{\sqrt{2}}
$$

and the minimum required value for $p_{1}$ in order to get smaller delay over the two-hop path is

$$
p_{1, \min }=p_{1 \mid p_{2}=0}=\frac{\sqrt{2}-1}{\sqrt{2}}=0.29
$$

b) Repeat the same calculation for the S-D pair in Fig. 2 .

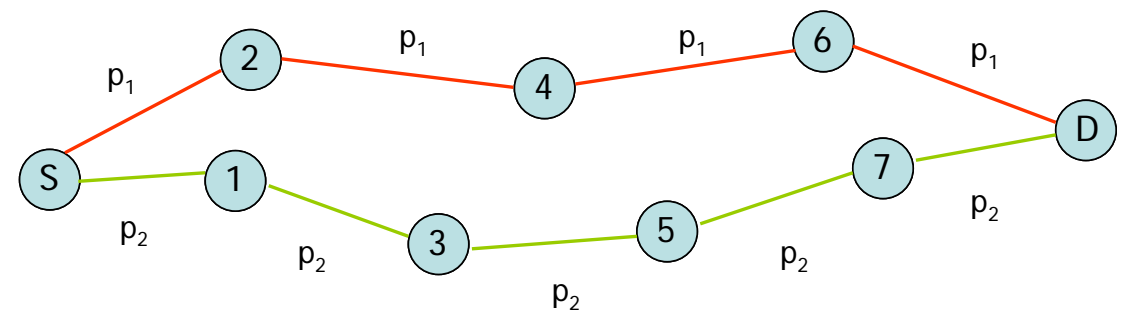

Fig. 2. Four-hop path vs. five-hop path

With the same rationale, the end-to-end normalized delays over paths S-2-4-6$\mathrm{D}$ and S-1-3-5-7-D are:

$$
D_{S 246 D}=\frac{4}{\left(1-p_{1}\right)^{2}} \text { and } D_{S 1357 D}=\frac{5}{\left(1-p_{2}\right)^{2}}
$$

Therefore,

$$
D_{S 246 D} \geq D_{S 1357 D} \Rightarrow \frac{4}{\left(1-p_{1}\right)^{2}} \geq \frac{5}{\left(1-p_{2}\right)^{2}} \Rightarrow \frac{\left(1-p_{2}\right)}{\left(1-p_{1}\right)} \geq \frac{\sqrt{5}}{2}=1.12 \Rightarrow p_{1} \geq \frac{0.12+p_{2}}{1.12}
$$

and the minimum required value for $p_{1}$ in order to get smaller delay over the two-hop path is

$$
p_{1, \min }=p_{1 \mid p_{2}=0} \approx 0.1
$$

Therefore, when there is no flexibility in increasing the transmit power or when this is not appealing due to energy constraints, there are scenarios where the rationale of the minimum-hop metric, as known from the wired networks, is can- 
celled. As the example suggests, it is more likely that the decisions made using the minimum hop count metric differ from the optimum ones along paths with many hops, since there the loss probability over the minimum hop links do not have to be as high as in paths with one or two hops. In any case, the message coming out of the example is that knowledge of the dynamically changing loss probabilities over the network links could support wiser routing decisions in wireless mesh networks.

This remark was made quite early by researchers in the field. What took more time and experimentation was the method to obtain this information from the network. Using the signal strength measurements to infer these loss probabilities was historically the first attempt in this direction.

\subsection{Signal strength based metrics}

Signal strength has been used as link quality metrics in several routing schemes for wireless mesh networks. The hypothesis is: since a packet is successfully received when the signal strength exceeds some threshold value, the signal strength could be viewed as a good indicator of the link quality. Nowadays, commodity wireless network adapters provide an average received signal strength value for every successfully received packet.

Signal strength values have been used in routing in two different ways:

- as control parameters for excluding routes with "bad" quality link from the route selection process.

- as conventional routing metrics, where some function of the signal strength is considered in the link(path) cost function

\subsubsection{Signal strength as control parameter for eliminating routes}

In [9] signal strength is measured upon packet reception passively. A preemptive region around a source is introduced and a path is considered likely to break when the power of the received packet becomes lower than a predefined preemptive threshold $P_{\text {threshold }}$. The threshold is defined as:

$$
P_{\text {threshold }}=\frac{P_{O}}{r_{\text {preemptive }}^{4}}
$$

where $P_{O}$ is a constant for each transmitter/receiver pair that depends on the antenna gain and height and $r_{\text {preemptive }}$ is the radius of the node's preemptive region. Receiver nodes generate a protocol specific warning message towards the source 
as soon as the reported signal strength of a received packet drops below $P_{\text {threshold }}$. Then, the source will search for a higher quality path to route its packets. Generally, more stable average values can be generated by having a number of message exchange rounds. The main disadvantage of the proposed preemptive routing mechanism is its assumption that links are symmetric. Since this does not often hold in reality (for example, see [10]), the proposed mechanism may suffer from instabilities. Nevertheless, the evaluation of the proposed method with simulations shows significant improvement in the performance of the AODV and DSR routing protocols, since the proposed modifications result in reduced number of broken paths.

In a similar approach, the Signal Stability-Based Adaptive Routing (SSAR) in [8] uses periodic link-layer beacons to get estimates of the link quality and forward route discovery packets only via routes involving stable links with good signal strength. The difference with the preemptive routing is that the decision to eliminate routes is not taken by the source but is rather distributed, with each node dropping packets from links with weak signal rather than issuing warning messages.

\subsubsection{Signal strength as routing metric}

Punnoose et al. [11] convert the signal strength into a link quality factor, which is then used to assign weights to the links. For a route consisting of $M$ hops, the link quality factor $L$ of the route is estimated as

$$
L=\prod_{s=1}^{M}\left(1-Q\left(\left(P_{\text {pred }_{i}}-P_{t h}\right) / \sigma\right)\right)
$$

where $Q(x)$ is the standard Q-function, $P_{\text {pred }_{i}}$ is the theoretically predicted power received by the $i^{\text {th }}$ node from the $(i-1)^{\text {th }}$ node, $P_{t h}$ is the receiving threshold and $\sigma$ is the variance of signal variations, which are assumed to be normally distributed.

The link quality factor is the product of probabilities computed for each hop that at a certain time in the future the signal level will be above the receiving threshold. The theoretically predicted power is calculated as follows: using linear position extrapolation based on the input data from GPS positioning and velocity information, estimates for the positions of all nodes one second in the future are calculated. These positions, along with some propagation model are used to obtain $P_{\text {pred }_{i}}$, while the default values for the variance of signal is $\sigma=6 \mathrm{~dB}$ and for the receiving threshold $P_{t h}=60 \mathrm{dBm}$.

\subsubsection{Correlation of signal strength with probability of successful packet delivery}



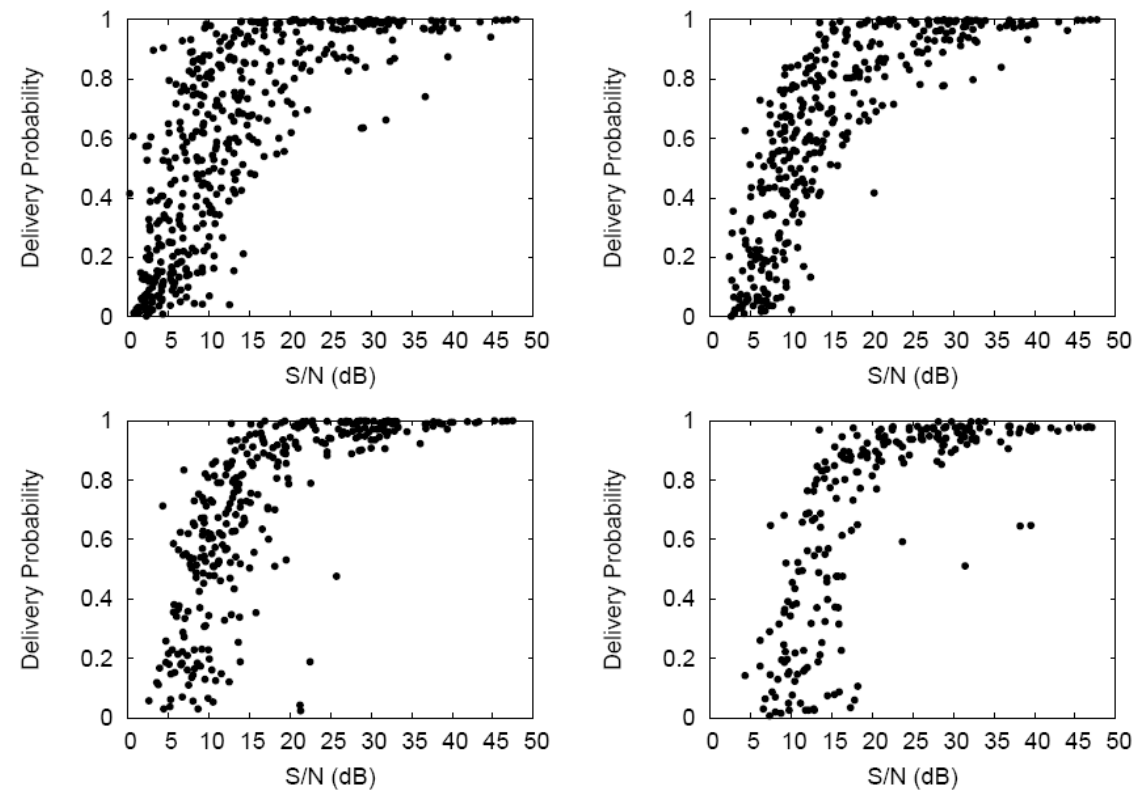

Fig. 3. Delivery probability at $1,2,5.5$, and $11 \mathrm{Mbit} / \mathrm{s}$ versus the averages $\mathrm{S} / \mathrm{N}$. Each data point represents an individual sender-receiver pair. Figure is adopted from [10].

Although correlation of signal strength and loss is used in the above metrics, whether such correlation exists is addressed in two studies.

In [12], the focus is on the packet delivery performance in sensor networks. It is reported that high signal strength implies low packet loss, however low signal strength does not necessarily imply high packet loss.

A similar observation is made in [10]. Link-level measurements in a wireless mesh network (Roofnet) demonstrate that although the signal strength values do affect the delivery probability, one cannot expect to use them as a predictive tool. This is clearly shown in Figure 3, which plots the link delivery probabilities at different rates versus the average $\mathrm{S} / \mathrm{N}$ (minimum signal-to-interference ratio for successful reception). Although the specification of the wireless card used in Roofnet suggests that the range of signal strength values for which the packet error rate would be between $10 \%$ and $90 \%$ (intermediate loss rates) is only $3 \mathrm{~dB}$ wide, the actual measured range of intermediate loss rates is much wider than that. Experiments using a hardware channel emulator demonstrate that an essential cause of intermediate loss rates is multipath fading due to reflections in the radio environment.

The results of both studies are aligned regarding the impact of the signal strength upon the delivery probability, but also the difficulty to get a mapping function between the two quantities. The signal strength, at least the values reported by most commercial 802.11 cards, represent coarse average values of the received signal strength and do not capture channel fading effects. Therefore, the 
signal strength does not lend itself to reliable estimates of the probability of packet loss over the network links, which, as discussed in section 3.1.1 could drive more intelligent routing decisions. An alternative method to obtain these probabilities is via active probe measurements.

\subsection{Active probing based metrics}

Inferring the probabilities of data loss in the network links via the signal strength values is one possibility; as discussed in section 3.2, the results were not very promising. The alternative approach is to carry out active measurements and use probe packets to directly estimate those probabilities.

Probing introduces various challenges. One concern with it is that it should be treated as normal traffic in the network, e.g., the packet sizes of probes should be equal to the actual traffic data so that what probes measure is as close to the target as possible. Likewise, probe packets should not be prioritized or treated preferentially in the network. On the other hand, if the probing packets are interlaced with the regular traffic (so-called intrusive or in-band measurement), the probes themselves influence the amount of traffic. Ferguson and Huston [13] compare this effect with the Heisenberg Uncertainty Principle. Lundgren et al. [14] and later Zhang et al. [15] observed that the different properties of unicast and broadcast communication in IEEE 802.11 systems may lead to similar effects: Probes that are sent using the broadcast mechanism will report neighbors that are not reachable using unicast communication. Both papers call this phenomenon the greyzone problem.

Even more important concern, in particular when wireless links are involved, is the overhead related to probe messages. The actual probing period is a tradeoff between measurement accuracy and signaling overhead.

Nevertheless, probing based approaches have proved promising in the context of wireless mesh networks. They measure directly the quantity of interest, rather than inferring it from indirect measurements, and do not rely on analytical assumptions. This is why these metrics have been particularly popular in the last five years. The main novelty came with the Expected Transmission Count (ETX) metric; then a whole family of metrics has emerged out of it that attempts to optimize routing performance under various assumptions for the link rates and the channels used in the network.

\subsubsection{Per-hop Round Trip Time (RTT)}

The per-hop Round-Trip Time (RTT) metric reflects the bidirectional delay on a link [16]. In order to measure the RTT, a probe carrying a timestamp is sent peri- 
odically to each neighboring node. Then each neighbor node returns the probe immediately. This probe response enables the sending node to calculate the RTT value. The path RTT metric is simply the addition of the link RTTs estimated over all links in the route. The RTT metric is a load-dependent metric, since it comprises queuing, channel contention, as well as 802.11 MAC retransmission delays. Besides the probe-related overhead, the disadvantage of RTT is that it can lead to route instability (phenomenon of self-interference).

\subsubsection{Per-hop packet pair delay (PktPair)}

The PktPair delay involves the periodic transmission of two probe packets backto-back, one small and one large, from each node. The neighbor node then measures the inter-probe arrival delay and reports it back to the sender. This technique is designed to overcome the problem of distortion of RTT measurements due to queuing delays. The PktPair metric is less susceptible to self-interference than the RTT metric, but it is not completely immune, as probe packets in multi-hop scenario contend for the wireless channel with data packets. To understand this, consider three nodes A, B and C in a chain where A sends data to C via B. Data packets sent to node B contend with probe packets of B destined to C. This increases the PktPair metric between B and C and consequently increases the metric along the path from A to C. Performance evaluation on an indoor wireless testbed showed that RTT performed 3 to 6 times worse than the minimum hop count, Packet Pair or ETX metrics in terms of TCP throughput [17]. As RTT is more sensitive to load, it performs worse than PktPair.

Both the RTT and PktPair metrics measure delay directly, hence they are loaddependent and prone to the self-interference phenomenon. Moreover, the measurement overhead they introduce is $O\left(n^{2}\right)$, where $n$ is the number of nodes. On the contrary, the metrics presented below are load-independent and the overhead they introduce is $O(n)$.

\subsubsection{Expected Transmission Count (ETX)}

The Expected Transmission Count (ETX) is one of the first routing metrics based on active probing measurements specifically designed for MANETs. Starting with the observation that minimum hop count is not optimal for wireless networks, De Couto et al. [18] proposed a metric that centers on bidirectional loss ratios. ETX estimates the number of transmissions (including retransmissions) required to send a packet over a link. Minimizing the number of transmissions does not only optimize the overall throughput, it does also minimize the total consumed energy if we assume constant transmission power levels, as well as the resulting interference in the network [19]. Let $d_{f}$ be the expected forward delivery ratio and $d_{r}$ be the re- 
verse delivery ratio, i.e., the probability that the acknowledgement packet is transmitted successfully. Then, the probability that a packet arrives and is acknowledged correctly is $d_{f} \cdot d_{r}$. Assuming that each attempt to transmit a packet is statistically independent from the precedent attempt, each transmission attempt can be considered a Bernoulli trial and the number of attempts till the packet is successfully received a Geometric variable, $\operatorname{Geom}\left(d_{f} \cdot d_{r}\right)$; therefore, the expected number of transmissions is:

$$
E T X=\frac{1}{d_{f} \cdot d_{r}}
$$

The delivery ratios are measured using link-layer broadcast probes, which are not acknowledged at the 802.11 MAC layer. Each node broadcasts a probe packet every second including in its probes the number of probes received from each neighboring node over the last $w$ seconds ( $w=10$ in their implementation). Each neighbor of a sender node A can then calculate the $d_{r}$ value to A each time it receives a probe from node $B$, as the ratio of the reported count over the maximum possible count $w$. The whole process is summarized in Fig. 4.

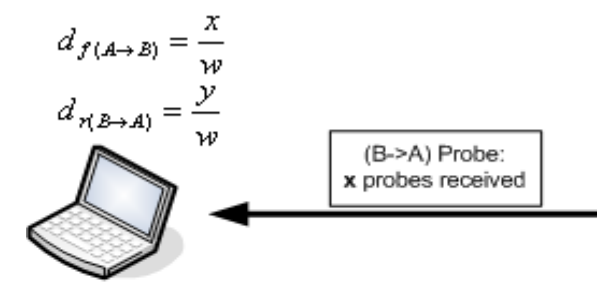

A

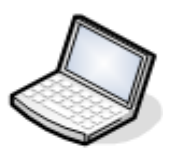

B

$$
E T X_{A}=\frac{1}{d_{r(B \rightarrow A)} * d_{f(A \rightarrow B)}}=\frac{1}{\left(\frac{y}{w}\right) *\left(\frac{x}{w}\right)}
$$

Fig. 4. ETX metric estimation for node A

Node B reports with the latest broadcast probe the number of probes $x$ received over the previous time window $w$. Node A estimates the probability that a data packet will be successfully transmitted to B in a single attempt. It also counts the number of probes $y$ received from node B over the same time and gets the ETX value for the link. The ETX along a path is defined as the sum of the metric values of the links that form this path.

The main advantages of the ETX metric are its independence from link load and its account for asymmetric links. In other words, ETX does not try to route around congested links and therefore it is immune to the phenomenon of self-interference. 
Measurements conducted on a static test-bed network show that ETX achieves up to two times higher throughput than minimal hop-count for long links. ETX is one of the few non hop-count metrics that has been implemented in practice in MANETs, e.g., as part of the OLSR protocol daemon (OLSRD) over multiple platforms [20].

The essential disadvantage of the ETX metric, as already mentioned earlier, is the overhead injected in the network from the probe packets. Furthermore, since broadcast packets are small and are sent at the lowest possible rate, the estimated packet loss may not be equal to the actual packet loss of larger data packets sent at higher rates. Moreover, it does not directly account for the link transmission rate; two links with different transmission rates, hence different transmission delays, may have the same packet loss rate. Finally, ETX is only relevant for radio interfaces that perform retransmissions.

\subsubsection{Expected Transmission Time (ETT), Medium Time Metric (MTM), and Weighted Cumulative Expected Transmission Time (WCETT)}

Draves et al. [21] observe that ETX does not perform optimally under certain circumstances. For example, ETX prefers heavily congested links to unloaded links, if the link-layer loss rate of congested links is smaller than on the unloaded links. Therefore, they address this proposing the Expected Transmission Time (ETT) metric incorporating the throughput into its calculation. Let $S$ be the size of the probing packet and B the measured bandwidth of a link, then the ETT of this link is defined as follows:

$$
E T T=E T X * \frac{S}{B}
$$

A similar metric, called Medium Time Metric (MTM), was independently proposed by Awerbuch et al. in [22]. The metric estimate for link $l$ and packet $p$ is a function of the link transmission rate, rate $(l)$, and the packet size, $\operatorname{size}(p)$, and is given by

$$
\tau(l, p)=\frac{\operatorname{overhead}(l)+\frac{\operatorname{size}(p)}{\operatorname{rate}(l)}}{\text { reliability }(l)}
$$

where the overhead(l) term accounts for the per-packet overhead of the link that includes control frames, back-off, and fixed headers, and the reliability $(l)$ term equals to the fraction of packets successfully delivered over the link. It is straightforward to see that there is an one-to-one correspondence between the terms $\operatorname{size}(p) \leftrightarrow S$, rate $(l) \leftrightarrow B$, and $E T X \leftrightarrow 1 /$ reliability $(l)$, as used in the equations 
describing the MTM and ETX metrics, respectively. The only difference between the two metrics lies in the explicit account for MAC-related overheads in the MTM metric; although, it seems that subsequent definitions of the ETT metric have also accounted for this term [23].

Draves et al. propose to use packet pairing techniques (see Section 3.3.2) to measure the transmission rate on each link at the expense of additional measurement overhead. On the contrary, Awerbuch et al. recommend the use of inter-layer communication, so that the routing layer can have access to relevant information and statistics maintained by the physical and MAC layer. This would require some standard interface that, at least for the moment, is not available on most wireless network adapter cards.

Draves et al. go one step further in their work to suggest computing the path metric as something more than just the sum of the metric values of the individual links in this path. Pure summation of link metrics does not take into account the fact that concatenated links interfere with each other, if they use the same channel. As many wireless technologies, including $802.11 \mathrm{a} / \mathrm{b} / \mathrm{g}$, provide multiple nonoverlapping channels, they propose an adaptation of the ETT metric accounting for the use of multiple channels, namely the Weighted Cumulative ETT (WCETT).

Let $k$ be the total number of channels of a system, the sum of transmission times over all hops on channel $j$ is defined as:

$$
X_{j}=\sum_{i \text { uses channell } j} \operatorname{ETT}_{i} \quad 1 \leq j \leq k
$$

As the total path throughput will be dominated by the bottleneck channel, which has the largest $X_{j}$, they propose to use a weighted average between the maximum value and the sum of all ETTs. This results in the formula:

$$
\text { WCETT }=(1-\beta) \cdot \sum_{i=1}^{n} E T T_{i}+\beta \cdot \max _{1 \leq j \leq k} X_{j}
$$

with $0 \leq \beta \leq 1$ being a tunable parameter. The authors describe different interpretation possibilities for this parameter. In their static test-bed implementation they showed that WCETT outperformed ETX by a factor of two and minimal hop count by a factor of four, when two different IEEE 802.11 radio cards per station were used. The main disadvantage of the WCETT metric is that it is not immediately clear if there is an algorithm that can compute the path with the lowest weight in polynomial or less time.

\subsubsection{Metric of Interference and Channel switching (MIC)}


The Metric of Interference and Channel switching (MIC) [24] improves WCETT by addressing the problem of intra-flow and inter-flow interference. The MIC metric of a path $p$ is defined as follows:

$$
\operatorname{MIC}(p)=\frac{1}{N \times \min (E T T)} \sum_{\text {linkl } l \in p} I R U_{l}+\sum_{\text {nodei } \in p} C S C_{i}
$$

where $N$ is the total number of nodes in the network and $\min (E T T)$ is the smallest ETT in the network, which can be estimated based on the lowest transmission rate of the wireless cards. The two components of MIC, IRU (Interference-aware Resource Usage) and CSC (Channel Switching Cost) are defined as:

$$
\begin{aligned}
I R U_{l} & =E T T_{l} \times N_{l} \\
\mathrm{CSC}_{i} & =\left\{\begin{array}{ll}
w_{1} & \text { if } C H(\operatorname{prev}(i)) \neq C H(i) \\
w_{2} & \text { if } C H(\operatorname{prev}(i))=C H(i)
\end{array} \quad 0 \leq w_{1} \leq w_{2}\right.
\end{aligned}
$$

where $N_{l}$ is the set of neighbors that the transmission on link $l$ interferes with, $\mathrm{CH}(i)$ represents the channel assigned for node $i$ 's transmission and prev(i) represents the previous hop of node $i$ along the path $p$.

The $I R U_{1}$ component copes for the inter-flow interference and corresponds to the aggregate channel time consumed (or the amount of bandwidth resource consumed) on a link $l$. In other words, this component includes the expected transmission time for an intended sender as well as the time neighbor nodes have to defer in CSMA/CA MAC protocols and favors a path that consumes less channel time at its neighboring nodes. The CSC component represents the intra-flow interference, favoring paths with more diversified channel assignments and penalizing paths with consecutive links using the same channel.

The MIC metric provides better performance because it considers intra/interflow interference and channel diversity. The disadvantage of the metric is the high overhead needed to estimate the per path $\mathrm{MIC}(\mathrm{p})$ value. Each node should be aware of the total number of nodes in the network; this in large networks may become very expensive.

\subsubsection{Multi-Channel Routing Metric (MCR)}

Kyasanur and Vaidya [25] extend WCETT in a different direction than MIC does; they take into account the cost of changing channels. Let InterfaceUsage $(i)$ be the fraction of time a switchable interface spends on transmitting on channel $i$ and let $p_{s}(j)$ be the probability that the used interface is on a different channel when we 
want to send a packet on channel $j$. If we assume that the total of the current interface idle time can potentially be used on channel $j$, we can estimate $p_{s}(j)$ as

$$
p_{s}(j)=\sum_{\forall i \neq j} \text { InterfaceUsage }(i)
$$

Let SwitchingDelay denote the switching latency of an interface. This value can be measured offline. Then, the cost of using channel $j$ is measured as

$$
S C\left(c_{i}\right)=p_{s}(j) * \text { SwitchingDelay }
$$

In order to prevent frequent channel switching of the chosen paths, a switching cost is included into the ETT metric, so that the resulting MCR metric becomes:

$$
M C R=(1-\beta) \cdot \sum_{i=1}^{n}\left(E T T_{i}+S C\left(c_{i}\right)\right)+\beta \cdot \max _{1 \leq j \leq k} X_{j}
$$

Simulation results evaluating the MCR metric have shown that network capacity can be improved by using multiple channels, even if only two interfaces per node are available.

\subsubsection{Modified ETX (mETX) and effective number of transmissions (ENT)}

Most of the ETX derivatives described so far in sections 3.3.4-3.3.7 expand the applicability of ETX into various directions not well captured by the original definition of the metric, such as the use of multiple channels that may interfere with each other, and the variation of link transmission rates and packet sizes. Nevertheless, all of them maintain at their core the estimator of successful delivery and expected number of transmissions, as it was coined in the original ETX proposal.

On the constrary, Koksal and Balakrishnan [26] focus exactly on the accuracy of the loss estimator function. The starting point is that, under certain conditions such as links with low average loss rate but high variability, the estimation capacity of the mean statistic is poor. They propose two alternative statistics for the estimation of required number of transmissions over a link.

- Modified ETX $(m E T X)$, is defined as $m E T X=\exp \left(\mu+\frac{1}{2} \sigma^{2}\right)$ with $\mu$ being the estimated average packet loss ratio of a link and $\sigma^{2}$ the variance of this value. Like ETX, mETX is additive over concatenated links.

- Effective Number of Transmissions (ENT), is defined as $E N T=\exp \left(\mu+2 \delta \sigma^{2}\right)$. The parameter $\delta$ acts as an additional degree of freedom with respect to $\mathrm{mETX}$; for $d=1 / 4$, ENT coincides with mETX. Its value depends on the number of subsequent retransmissions, which will cause the link layer protocol to give up a transmission attempt. 
Empirical observations of a wireless mesh network suggest that mETX and ENT rate could achieve a $50 \%$ reduction in the average packet loss, when compared with ETX.

\subsection{Mobility-aware metrics}

The metrics that are based on active measurements with probe packets, such as the ETX and its derivatives described in sections 3.3.3-3.3.7 outperform the hop count metric in static networks. The situation appears to be reversed in mobile scenarios. As nodes move around, links may come up and down altering the optimal routes in the network. Metrics relying on measurements need some time to update their estimate of the link quality and this may result in significant performance degradation, in particular when routes change multiple times within the duration of the data transfer. On the contrary, the minimum-hop count metric can use the new links almost as quickly as they become available [17].

Mobility-aware metrics aim at the selection of routes with higher expected lifetime to minimize the routing overhead related to route changes and their impact on throughput. The metrics largely use signal strength measurements and their rate of variation to infer the stability of links and routes. The path average degree of association stability, as proposed in the context of associativity-based routing (ABR) in [7], and the affinity metric defined in [27] and reused by the Route-Lifetime Assessment Based Routing (RABR) protocol in [28], are example metrics of this category.

\subsubsection{Link associativity ticks and path average degree of association stability}

Mobile nodes transmit link-layer beacons at fixed time intervals (default value: one sec) and measure the received number of probs (associativity ticks) from their neighbors. These values serve as indicators of the actual stability of the link. Low values of associativity ticks imply mobile nodes in high mobility state, whereas high associativity ticks, beyond some threshold value $A_{t h r}$, are obtained when a mobile node is more stable. The underlying assumption of the metric is that nodes alternate between periods of transition/migration and idleness.

The average degree of association stability over route $R, A_{\text {ave }}^{R}$, is estimated as a function of the associativity ticks over all links along the route

$$
A_{\text {ave }}^{R}=\frac{1}{n} \sum_{l \in R} \mathbf{1}_{A_{l} \geq A_{\text {thr }}}
$$

where $\mathbf{1}$ is the logical indicator function and $n$ is the number of links in route $R$. In associativity-based routing, the routes considered for selection are only those with relay load lower than some threshold. The selected route is simply the one with 
the highest average degree of association stability. In case two routes feature the same average degree of association stability, the route with the minimum hop count is selected.

\subsubsection{Link affinity and path stability}

The link affinity is an estimator of the link lifetime. The affinity of a link $l_{e}$ is related to the received power over that link $P_{e}$, its rate of change, and a threshold $P_{T H R}$, determining whether the link is broken or not. Each node samples periodically, every interval $d t$, the strength of the signal received over $l_{e}$. Defining the signal strength change rate as $\Delta P=\left(P_{e}\right.$ (current) $-P_{e}$ (previous $\left.)\right) / d t$ and the average rate of signal strength change as $\Delta P_{e}^{a v e}$, the link affinity is determined by:

$$
a_{e}=\left\{\begin{array}{l}
\text { high, if } \Delta \mathrm{P}_{\text {ave }}>0 \\
\left(P_{\text {THR }}-P_{e}\right) / \Delta P_{e}^{a v e}, \text { if } \Delta \mathrm{P}_{\text {ave }}<0
\end{array}\right\}
$$

The affinity between two nodes $\mathrm{A}$ and $\mathrm{B}$ is then given by:

$$
\eta_{A B}=\min \left[a_{A B}, a_{B A}\right]
$$

The route stability is then given by the minimum of the affinities of all links lying in the route

$$
\eta_{R}=\min _{l \in R} n_{l}
$$

The route is selected as long as the estimated value for its stability exceeds the required time to transfer data, whose estimate equals the time required to transmit data over the link capacity $C$. A correction factor $f$ accounts for the imprecision of the metric, so that the check performed for the route is:

$$
D_{A B} / C \stackrel{?}{<} f \cdot \eta_{A B}
$$

If the inequality holds, the route $R$ is selected. Otherwise, the next available path, if it exists.

In both aforementioned approaches, the link metrics are piggybacked on the route discovery packets that propagate from the source towards the destination. The decision upon the route selection is taken at the receiver.

\subsubsection{Mobility-model driven metrics}

Mcdonald and Znati [29] propose another routing metric, which defines a probabilistic measure of the availability of links that are subject to link failures caused by node mobility. They base their considerations on a random walk model. Each node is characterized by three values that describe the statistical distribution of the mean and variance of the speed of a node as well as an average interval time. To- 
gether with an estimated communication radius, Mcdonald and Znati derive a sophisticated function, which estimates the expected availability of a link.

Various other metrics were proposed, based on other mobility models. Among them are the metrics described by Gerharz et al. [30] and Jiang et al. [31] that estimate the average residual lifetime of a link. However, the weak link in all these studies is the assumption that all nodes have similar mobility characteristics. In mesh networks, this obviously is not the case.

\subsection{Energy-aware metrics}

In contrast with wired networks, energy consumption may represent an essential constraint in wireless mesh networks. Sensors as well as small and batteryoperated wireless devices have restricted battery lifetime and are most vulnerable to the energy constraints. Energy-related objectives are often at odds with performance related objectives. For example, choosing paths so that the overall delay(throughput) is minimized may result in overuse of certain nodes in the network and premature exhaustion of their battery. Therefore, energy concerns have to be properly reflected in the definition of routing metrics.

The total energy consumed when sending and receiving a packet is influenced by various factors such as the wireless radio propagation environment, interference from simultaneous transmissions, MAC protocol operation, and routing algorithm. Unsuccessful reception due to interference (external, inter-flow or intraflow interference) results in retransmissions and higher energy consumption. The essential objectives of routing metrics targeting at minimizing energy consumption are then: i) to minimize overall energy consumption and ii) to maximize the time until the first node runs out of energy.

\subsubsection{Minimal Total Power routing (MTPR)}

One of the first proposals in energy related routing metrics is to minimize the per packet consumed energy. The rationale of the metric, called Minimal Total Power Routing metric MTPR in [32], is that this way the overall energy consumption is minimized. Singh et al. [35] formalize this idea as follows: let $e_{i, j}$ denote the energy consumed for transferring a packet from node $i$ to the neighboring node $j$. Then, if the packet has to traverse the path $p$, including nodes $n_{1}, \ldots, n_{k}$, the total energy $\mathrm{E}$ required for the packet transfer is

$$
E=\sum_{i=1}^{k-1} e_{n_{i}, n_{i+1}}
$$


Out of the full set $P$ of possible paths, the route $p^{\prime}$ that minimizes total energy is selected

$$
p^{\prime}=\left\{p \in P \mid E^{p}<E^{q}, \forall q \in P\right\}
$$

Interestingly, when considering lightly loaded paths and good links, the MTPR metric tends to yield the same route with the minimum hop-count metric. In those cases, both the overall delay and the energy consumption are proportional to the hop count of the path; hence minimizing the one is equivalent to minimizing the other. The situation changes when we consider error-prone or high-contention links, where more than one transmission attempts are required to get the packet through. Then, the MTPR may select a different route resulting in higher hop count; this is similar to what the ETX metric and its derivatives do, as discussed in section 3.3.

A disadvantage of this packet-oriented metric is that it does not directly take into account the nodes' remaining battery lifetimes. It is quite probable that seeking for routes that minimize the per-packet energy consumption, one might end up with nodes that forward traffic from multiple concurrent flows and consume their battery power much faster than other nodes.

\subsubsection{Minimum battery cost routing (MBCR)}

In order to address the aforementioned problem and balance the energy consumption over all nodes in a network, the battery capacity of a node is taken into consideration in the routing metric definition. The "Minimum Battery Cost Routing" (MBCR) [33] is based on the remaining battery capacity of the node. The ratio of battery capacity $R_{b r c}$ is defined as

$$
R_{b r c}=\frac{E_{i}}{E_{\max }}=\frac{\text { Battery remaining capacity }}{\text { Battery full capacity }}
$$

Under the assumption that all nodes have the same battery full capacity, a cost value $f_{i}\left(E_{i}\right)$ is assigned to each node $n_{i}$ based on its residual battery capacity $E_{i}$ :

$$
f_{i}\left(E_{i}\right)=\frac{1}{E_{i}}
$$

Then the total available battery lifetime along a path $p$ is the sum of the battery capacities of all nodes along the route

$$
R_{b c r}^{p}=\sum_{n_{i} \in p} f_{i}\left(E_{i}\right)
$$

Out of the full set $P$ of possible paths, the one selected, $p^{\prime}$, features minimum total residual battery capacity

$$
p^{\prime}=\left\{p \in P \mid R_{b c r}^{p}>R_{b c r}^{q}, \forall q \in P\right\}
$$

The apparent disadvantage of MBCR is that the selected route may well feature individual nodes with small remaining battery capacity. In Figure 5, for example, 
path 1 will be selected even though the remaining battery capacity cost value $f_{i}\left(E_{i}\right)$ of node 3 is very low $\left(f_{3}=90\right)$. To address this problem, a classification of nodes in three categories based on the cost value $f_{i}\left(E_{i}\right)$ is proposed in [34]. The first category consists of nodes with less than $10 \%$ of their initial battery capacity. The routing algorithm in this case avoids paths with nodes of this category, as long as there is an alternative path. The second category includes nodes that their remaining energy is between 10-20\% of their initial energy. This signifies that the nodes are running out of energy and the routing algorithm should also avoid them if possible. Otherwise, a node is not treated specially. Referring to the example illustrated in Figure 5, path 2 would have been selected. Simulation evaluation showed an increase of nodes' lifetimes of up to $65 \%$ under low-traffic and up to $25 \%$ under heavy-traffic scenarios.

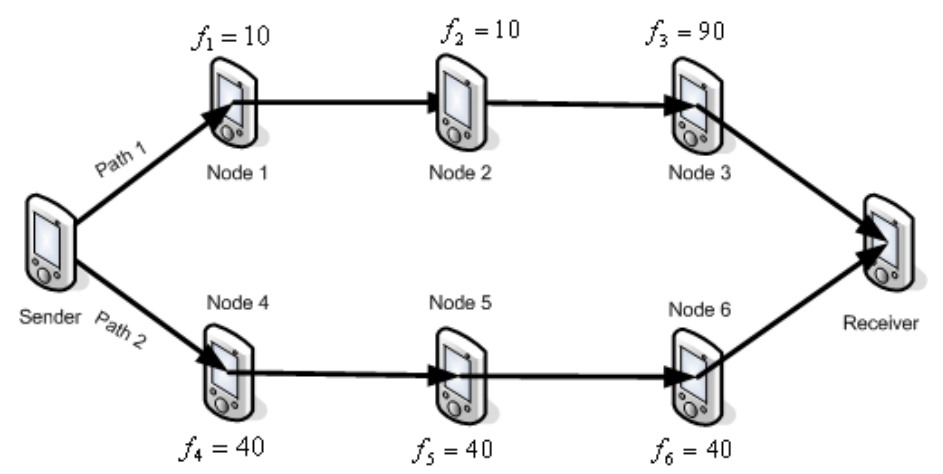

Fig. 5. Route selection based on energy cost value. Route 1 is selected over route 2 , although it involved one node (node 3 ) with very low residual battery capacity.

\subsubsection{Min-Max Battery Cost Routing (MMBCR)}

The Min-Max Battery Cost Routing (MMBCR) metric [35] addresses more explicitly the shortcoming of the original MCBR metric in avoiding nodes with very low residual battery capacity along paths with high overall battery capacity. The idea is to select a path, which minimizes the maximum power required at any node in a network. In agreement with the formulation in section 3.5.2, with MMCBR the chosen path $p^{\prime}$ must fulfill

$$
p^{\prime}=\min _{p \in P} \max _{n_{i} \in p} f_{i}\left(E_{i}\right)
$$

Simulation results showed significant reduction of energy consumption by using shortest-cost routing as opposed to shorted-path routing. However, there is no guarantee that the MMBCR minimizes the total energy consumed over the path, 
making clear a trade-off between individual node and overall system energy optimization.

\subsubsection{Conditional max-min battery capacity routing (CMMBCR)}

Toh in [36] merges MTPR and MMBCR into one single hybrid routing metric called Conditional Max-Min Battery Capacity Routing (CMMBCR) metric. Firstly, CMMBCR searches paths using MTPR, with the restriction that all nodes need to have a remaining percentage battery capacity that exceeds a threshold value $\gamma$. If there is no such path, MMBCR is used.

The threshold $\gamma$ effectively operates as a tuning knob that can shape the behavior of the metric towards the one or the other metric; when $\gamma=0$, the CMMBCR degenerates to the MTPR metric, whereas for $\gamma=100$ CMMBCR behaves like the MMBCR metric.

Kim et al. [37] compare MTPR, MMBCR and CMMBCR. Their first finding was that overhearing the transmissions of some neighboring nodes does have a significant impact on the performance of each metric and all behave similarly. In dense networks MTPR allows connections to live longer, whereas in sparse networks where it is more important to avoid network partition MMBCR performs better.

\subsubsection{Maximal residual energy path routing (MREP)}

Chang and Tassiulas in [38] propose a link metric that takes into account the remaining battery capacity and the necessary transmission energy for their Maximum Residual Energy Path (MREP) algorithm. Let $e_{i, j}$ be the energy consumed to send one packet over the link from node $i$ to node $j, E_{j}$ the initial battery energy (capacity), and $\underline{E}_{j}$ the residual energy at node $j$. Chang and Tassiulas define two metrics for the $i \rightarrow j$ link: The remaining energy $d_{i, j}$ of a node, defined as

$$
d_{i, j}=\frac{1}{\underline{E}_{j}-e_{i, j}},
$$

and the inverse of the residual capacity of a node in terms of packets that can be delivered with the remaining energy:

$$
d_{i, j}=\frac{e_{i, j}}{\underline{E}_{j}}
$$

Performance evaluation with simulations in scenarios with highly mobility, both metrics came quite close to a theoretically predicted average node lifetime (theoretical values are calculated using linear programming). Refining their work in [39], they propose a more general formula: 


$$
d_{i, j}=e_{i, j}^{x_{i}} \underline{E}_{i}^{-x_{2}} E_{i}^{x_{3}}
$$

where $x_{1}, x_{2}$ and $x_{3}$ are nonnegative weighting parameters. Simulation evaluation reveal that with reasonable setting of the parameters, the theoretical maximal lifetime, the worst-case lifetime, and the transfer reliability can be well approximated.

\subsubsection{Power and Interference based (PIM) metric}

Michail and Ephremides in [40] study the problem of energy-efficient routing in a more concrete context, namely that of connection-oriented traffic. Every node avails one or more radio interfaces and can make use of a set of $k$ frequency channels to communicate with its neighbors. The authors incorporate interference by considering that each transmission blocks certain hops (sender-destination node pairs) and seek to minimize both the transmission power required for the communication of nodes $i$ and $j$ and the number of blocked hops (links)

$$
C_{i, j}=\left\{\begin{array}{l}
\frac{P_{i, j}}{P_{\max }}+\frac{\left|B_{i, j}\right|}{|B|}, \text { if } \sum_{k=1}^{m} f^{(i, j)}(k)>0 \\
\infty
\end{array}\right.
$$

where $c_{i, j}$ is the cost of a link from node $i$ to $j, P_{i, j}$ is the power needed for successful transmission, $P_{\max }$ is the maximum transmission power, $\left|B_{i, j}\right|$ is the number of blocked links from $i$ to $j$ and $|B|$ is the overall number of links in the network. The metric gets a finite value as long as there is at least one frequency channel available for communication between the two nodes.

Their metric is called Power and Interference-based Metric (PIM). Its performance evaluation is carried out with simulation but is limited to a comparison with another metric considered in the same paper, the minimum power metric MPM, which only considers the energy consumed in each transmission $\left(c_{i, j}=P_{i, j}\right)$. The results show that PIM outperforms MPM in terms of energy consumption, while achieving better fairness in terms of energy expenditure per node.

\subsection{Routing metrics in standardization arena}

Standardization work with respect to Wireless multihop Networks is carried out primarily within the IEEE, as part of the standardization work on various aspects of the $802.11 \mathrm{x}$ family of protocols. The respective working group is the $802.11 \mathrm{~s}$, which, as the time of writing, is working on an IEEE 802.11s standard specifica- 
tion [41]. The standard addresses routing recommending the use of the Airtime Link metric as the default routing metric. The metric is a measure of the amount of consumed channel resources for transmitting a frame over a particular link. The airtime cost $C_{a}$ for each link is calculated according to the following formula:

$$
c_{a}=\left[O_{c a}+O_{p}+\frac{B_{t}}{r}\right] \cdot \frac{1}{1-e_{f r}}
$$

where $O_{c a}$ is the channel access overhead, $O_{p}$ the MAC protocol overhead, $B_{t}$ the number of bits of a constant test frame depending on the IEEE 802.11 transmission technology, $r$ the transmission bit rate in Mbit/s on the current conditions with frame error rate $e_{f r}$. Interestingly, the Airtime metric definition points directly to the MTM and the ETT metrics described in section 3.3.4.

The Airtime Link Metric parameters for the two main IEEE 802.11 physical layers are listed in Table 1.

Table 1 Airtime Link Metric constants

\begin{tabular}{lll}
\hline Parameter & $802.11 \mathrm{a}$ & $802.11 \mathrm{~b}$ \\
\hline Channel Access Overhead: $O_{c a}$ & $75 \mathrm{~s}$ & $335 \mathrm{~s}$ \\
Protocol Overhead: $O_{p}$ & $110 \mathrm{~s}$ & $364 \mathrm{~s}$ \\
Number of bits in test frame: $B_{t}$ & 8224 & 8224 \\
\hline
\end{tabular}

Standardization work within the Internet Engineering Task Force (IETF) has mainly focused on the Mobile Ad Hoc Networks (MANETs); the homonymous working group (WG) has issued various RFCs on routing protocols such as AODV (RFC 3561), OLSR (RFC 3626), and DSR (RFC 4728). Work on network performance metrics is carried out within the ippm WG. Although the work is quite general and does not focus on routing, there are several RFCs addressing practical aspects of measurements that have direct application to the area of routing metrics as well. Examples are the RFC 2680 on one-way packet loss, and RFC 2681 on round-trip delay.

\section{Taxonomy}

In Table 2, a classification of the routing metrics based on the following criteria is illustrated: i) optimization objectives, ii) the method used to acquire the needed information to compute the metric and iii) the function used to compute the metric along a path. 


\begin{tabular}{|c|c|c|c|}
\hline Metrics & $\begin{array}{l}\text { Optimization } \\
\text { objectives }\end{array}$ & $\begin{array}{l}\text { Metric Computation } \\
\text { Method }\end{array}$ & $\begin{array}{l}\text { Path metric } \\
\text { function }\end{array}$ \\
\hline 1. Hop Count & - Minimize delay & $\begin{array}{l}\text { - Use of locally available } \\
\text { information }\end{array}$ & - Summation \\
\hline $\begin{array}{l}\text { Signal Strength based: } \\
\text { 1. Preemptive routing [9] } \\
\text { 2. SSAR [8] } \\
\text { 3. Link quality factor[11] }\end{array}$ & $\begin{array}{l}\text { - Higher expected route } \\
\text { lifetime }\end{array}$ & $\begin{array}{l}\text { - Use of locally available } \\
\text { information }\end{array}$ & $\begin{array}{l}\text { - Not defined, } \\
\text { routing algorithm } \\
\text { decision. }\end{array}$ \\
\hline $\begin{array}{l}\text { Active Probing: } \\
\text { 1. Per hop RTT [17] } \\
\text { 2. Per hop PktPair [17] } \\
\text { 3. ETX [18] } \\
\text { 4. ETT [21] } \\
\text { 5. MTM [22] } \\
\text { 6. WCETT [21] } \\
\text { 7. MCR [25] } \\
\text { 8. Modified ETX [26] } \\
\text { 9. ENT [26] } \\
\text { 10. MIC [24] }\end{array}$ & $\begin{array}{l}\text { - Minimize delay } \\
\text { - Maximize probability } \\
\text { of data delivery } \\
\end{array}$ & - Active probing & Summation \\
\hline $\begin{array}{l}\text { Mobility aware: } \\
\text { 1. ABR [7] } \\
\text { 2. Link affinity metric [27] }\end{array}$ & $\begin{array}{l}\text { - Higher expected route } \\
\text { lifetime }\end{array}$ & $\begin{array}{l}\text { - Active probing } \\
\text { - Metrics piggybacked to } \\
\text { route discovery packets }\end{array}$ & $\begin{array}{l}\text { - Not defined, } \\
\text { routing algorithm } \\
\text { decision. }\end{array}$ \\
\hline $\begin{array}{l}\text { Energy-aware: } \\
\text { 1. MTPR [32] } \\
\text { 2. MBCR [33] } \\
\text { 3. CMMBCR [36] } \\
\text { 4. MREP [38] } \\
\text { 5. PIM [40] } \\
\text { 6. MMBCR [35] }\end{array}$ & $\begin{array}{l}\text { - Minimize energy } \\
\text { consumption }\end{array}$ & $\begin{array}{l}\text { - Use of locally available } \\
\text { information }\end{array}$ & $\begin{array}{l}\text { - Summation } \\
\\
\text { 3- Order statistics } \\
\text { (Min-max) }\end{array}$ \\
\hline $\begin{array}{l}\text { Standardization: } \\
\text { 1. AirTime [41] }\end{array}$ & - Minimize delay & $\begin{array}{l}\text { - Active probing } \\
\text { - Use of locally available } \\
\text { information }\end{array}$ & $\begin{array}{l}\text { - Not defined, } \\
\text { routing algorithm } \\
\text { decision. }\end{array}$ \\
\hline
\end{tabular}

Table 2. Taxonomy of routing metrics.

${ }^{2}$ The routing algorithm disseminates the information needed to calculate the metric. 


\section{Thoughts for practitioners}

The presentation of routing metrics and their inline discussion lend themselves to several conclusions that could be of interest to wireless mesh networking practitioners:

- There is no "one size fits all" solution for routing in wireless mesh networking. This is no surprise, since the principle applies to many different areas of network engineering. There is a great variety of protocols, which have been proposed with different applications and priorities in mind. For example, energy-aware metrics are more appropriate for sensor networks or low-end, battery-powered devices that must operate without access to electricity grid for large intervals of time. Metrics that rely on active probing appear to have superior performance well in static wireless mesh networks. On the contrary, in high-mobility scenarios, mobility-aware metrics may result in selection of better routes.

- Simplicity does not always pay off. Shortest-path routing has seen enormous success in wired networks, primarily due to its simplicity. The combined dynamics of the wireless radio propagation, interference, node mobility, and, where relevant, energy constraints result in error-prone links and highly dynamic network topology, making the routing task much more challenging. The minimum hop count metric is not adequate in these cases, if optimum performance is sought after.

- There are multiple tradeoffs amongst routing metrics, even when their objectives are identical. The result is high flexibility at network configuration level and the possibility to tailor the routing behavior to the requirements and constraints of a particular network scenario. For example, link loss estimation accuracy can be traded with control data overhead; and network/system battery lifetime can be compromised with optimum application performance.

- There is a large margin for improvement of current network card hardware. What seems to be lacking at the moment is a clean interface between the network/routing layer and the lower layers of the radio interface, which would allow to take advantage of all the information and state maintained at the lower radio layers. Proposed routing metrics based on signal strength appear to be hard constrained by the limited monitoring and reporting capabilities of the network cards. 


\section{Directions for future research}

There are several issues with respect to routing metrics for wireless mesh networks that could benefit from further research. We discuss two of them, which in our opinion are the most important ones.

Multiple access interference has always been one of the main concerns when building wireless networks. Whereas its impact is quite well understood and addressed in infrastructure-based cellular networks, its characteristics and impact in wireless mesh networks are less well understood. There is consensus in the research community that the level of interference should be an input for routing protocols. Most routing metrics that have been proposed to overcome the inefficiencies of minimum hop count routing rely on active probing methods to drive routing decisions. The main disadvantages of these approaches are that they impose additional overhead and they suffer from inaccuracy and responsiveness to network node mobility. However, none of these metrics capture the impact of interference explicitly. In fact, it is not even clear how to best measure interference [23]. Interference-aware routing can avoid the pitfalls of the measurement-based approaches, and is an open area of research that could result in improvements in path metric computation and consequently in route selection.

Currently the $802.11 x$ suite of standards does not provide much information to higher layers. The only channel quality measure reported from commodity wireless adapters is the "Received Signal Strength Indicator" (RSSI) value which is also vendor-dependent. However, standardization efforts within IEEE 802.11 are preparing standards (802.11k [42] for wireless LANs and 802.11s [41] for wireless mesh networks), which will enable higher layers to obtain detailed channel condition information from the PHY and the MAC layers and provide additional flexibility with respect to transmit power control. These standards will include signal strength measurements and neighbor reports containing information on neighboring nodes as well as link quality metrics such as the Airtime metric. The use of this information to develop more sophisticated and efficient routing metrics is expected to be an area for future research.

\section{Conclusions}

This chapter presented an overview of routing metrics specifically designed for wireless mesh networks. Whereas in wired networks the hop count metric remains the most attractive solution, in wireless mesh networks interference, link asymmetry, mobility, and energy-related considerations represent additional challenges that require more elaborate solutions. The proposed routing metrics address partially the aforementioned issues considering different optimization objectives and applying various techniques such as signal strength measurements, active probing, 
energy consumption monitoring, and prediction of link breakage due to node mobility. Further improvement over what is achievable today is expected by better understanding the impact of interference and the exploitation of MAC and PHY layer information that will be available from commodity wireless adapters in the future.

\section{Terminology/Keywords}

1. Routing metric: A routing metric is a value assigned by a routing algorithm and used to determine whether one route performs better than another.

2. Hop count: Hop count is the number of wireless links (hops) traversed by a packet between its source and destination.

3. Minimum hop count: The minimum number of "hop" among all available paths between a source and a destination (shortest route).

4. Link metric: A value quantifying the quality of a link. This value is used by the routing algorithm to determine a route between a source and a destination.

5. Path cost function: A function to derive the path metric from the individual link metric values estimated for each link in the path.

6. Path metric: The metric of a path derived from the path cost function.

7. Active probe measurement: A method of measuring the properties of a link/path, whereby special packets (probes) are generated and sent periodically in order to infer properties of a link.

8. ETX: Popular active probe measurement metric predicting the number of retransmissions required to deliver a packet all the way to its destination.

9. Self-interference: Once a link is recognized as good, it is chosen by the routing protocol and starts getting used till is overloaded and is assigned with a worse metric value. As traffic starts to route around this link, its metric value increases again and the effects starts anew. The phenomenon of self-interference results in route oscillations.

10.AirTime Link metric: A measure of the amount of consumed channel resources for transmitting a frame over a particular link. Recommended metric within the forthcoming 802.11s standard.

\section{Review Questions}

1. Mention possible relations between link metrics and the respective path metrics. Give one example of path metrics that results from the summation and another that results from the multiplication of link metrics. 
- The answer lies in section 2.2 and, for examples one should consider section 3 . Summation, multiplication, statistics such as the min, max, mean of the link metrics are all relevant to the derivation of the path metric out of link metrics. An example of additive metric is the expected transmission count metric. An example of multiplicative metric is the probability of successful delivery.

2. Which are the four ways used generally by (Wireless Multihop) network nodes to obtain the information they require for the computation of the actual routing metric value? List them in increasing order of control traffic overhead they generate.

- The answer lies in section 2.3. The four ways, in increasing order of control traffic overhead they generate are: reuse of local information, passive measurements (neither of the two creates additional control data traffic), piggyback probing, active probing.

3. What is the self-interference phenomenon when we refer to routing metrics?

- The answer is in section 2.3. The phenomenon refers to the oscillation phenomena appearing in the route selection process as a result of frequent changes in the values of route metrics and the resulting decisions of the routing protocols.

4. Explain why the minimum hop count routing metric does not always yield minimum delay paths in wireless multi-hop networks.

- The answer lies in section 3.1.1, with example 1 illustrating the argument with simple scenarios. In summary, due to the non error-free wireless links, the delay is a function of the total transmissions (retransmissions) required over the path links. Depending on the actual loss rates encountered in those links, the route selected by the minimum-hop count metric may result in higher end-toend delay than a route with higher-than-minimum number of hops.

5. Why is the ETX metric less prone to the self-interference phenomenon than the RTT metric and the PktPair Delay metric?

- The answer is spread in section 3.3 Both the RTT and PktPair delay metric measure delay and are highly load dependent. ETX is load-independent.

6. What are the disadvantages of the ETX metric?

- The answer is in section 3.3.1. 
- Since probe packets are small, they may not experience the same loss rate with data packets of larger size, i.e., they might underestimate or overestimate the achievable throughput.

- Probe packets are sent at the lowest possible rate $(1 \mathrm{Mbit} / \mathrm{sec}$ in $802.11 \mathrm{~b} / \mathrm{g}$ and $6 \mathrm{Mbit} / \mathrm{sec}$ in 802.11a); the metric does not account for the transmission rates of the links within a path.

- As ETX is not load-dependent, a heavily loaded link may be selected if it has low loss rate.

7. What drawbacks of ETX does each of its derivatives address (ETT, WCETT, mETX)

- The answer is spread over sections 3.3.2-3.3.4. ETT weights the ETX metric with the expected packet transmission time, so that the impact of the link transmission rates is accounted for. WCETT considers the use of multiple, nonoverlapping channels along the path and promotes routes that feature minimum co-channel interference. Modified ETX...

8. Describe the main trade-off introduced by the Minimal Total Power (MTPR) and the Min-Max Battery Cost Routing (MMBCR) metric. How does the Conditional max-min battery capacity routing (CMMBCR) metric combine properties of the two metrics?

- The answer is coming out of sections 3.5.1. 3.5.3, and 3.5.4. MTPR gives priority to the minimization of the total battery capacity consumption per packet, potentially at the penalty of power-draining some busy nodes faster than others. MMBCR, on the other hand, circumvents paths containing nodes with low batter capacity, potentially at the expense of higher energy consumption per packet. Finally, CMMBCR introduces a control parameter, which allows tuning the behavior of the metric closer to one of the two metrics.

9. Why active probe measurements are not suitable for mobility-aware metrics?

- The answer is in section 3.4. Active probe methods require some time to draw good and stable link quality estimation. In case of frequent link breakages, this method would give incorrect metric estimations.

10. What is the recommended routing metric in the IEEE 802.11s forthcoming standard? Which one of the reviewed routing metrics does it resemble?

- The answer is in section 3.7. The recommended metric is called Airtime Link metric; it is almost identical to the Medium Time Metric (MTM) and the Expected Transmission Time (ETT) metric. 


\section{References}

1. G. Apostolopoulos, R. Guerin, S. Kamat, S.K. Tripathi, Quality of Service Based Routing: A Performance Perspective, ACM SIGCOMM, October 1998

2. A. Khanna and J. Zinky. The revised arpanet routing metric. SIGCOMM Comput. Commun. Rev., vol. 19, no. 4, pp. 45-56, 1989.

3. T. Clausen and P. Jacquet. Optimized link state routing protocol (OLSR). IETF RFC 3626, October 2003; http://www.ietf.org/rfc/rfc3626.txt.

4. D. Johnson and D. Maltz. Dynamic source routing in ad hoc wireless networks. In T. Imielinski and H. Korth, editors, Mobile Computing, Dordrecht, The Netherlands, 1996. Kluwer Academic Publishers.

5. C. Perkins and P. Bhagwat. Highly dynamic destination-sequenced distance-vector routing (dsdv) for mobile computers. In Proceedings of SIGCOMM, October 1994.

6. C.E. Perkins and E.M. Royer. Ad hoc on-demand distance vector routing. In Proceedings of the 2nd IEEE Workshop on Mobile Computing Systems and Applications, pp. 90-100, New Orleans, LA, February 1999.

7. C-K. Toh. Associativity-based routing for ad hoc mobile networks. Wireless Personal Communications, vol. 4, no. 2, pp. 103-139, March 1997.

8. R. Dube, C. Rais, K. Wang, and S. Tripathi. Signal stability based adaptive routing (SSA) for ad hoc mobile networks. in IEEE Personal Communication, February 1997.

9. T.Goff, N. Abu-Aahazaleh, D. Phatak, and R. Kahvecioglu. Preemptive routing in ad hoc networks. In Proceedings of MOBICOM ‘01, 2001.

10. D. Aguayo, J. Bicket, S. Biswas, G. Judd, R. Morris. Link-level measurements from an 802.11b mesh network, In Proceedings of SIGCOMM 2004.

11. R. Punnose, P. Nitkin, J. Borch, and D. Stancil. Optimizing wireless network protocols using real time predictive propagation modeling. In RAWCON, Aug 1999.

12. J. Zhao and R. Govindan. Understanding packet delivery performance in dense wireless sensor networks. In SenSys, Nov. 2003.

13. P. Ferguson and G. Huston. Quality of Service - Delivering QoS on the Internet in Corporate Networks. Robert Ipsen / John Wiley \& Sons, Inc., 1998.

14. H. Lundgren, E. Nordström, and C. Tschudin. Coping with communication gray zones in ieee 802.11b based ad hoc networks. In Proceedings of WOWMOM '02. New York, NY, USA, pp. 49-55, 2002.

15. H. Zhang, A. Arora, and P. Sinha. Learn on the fly: Data-driven link estimation and routing in sensor network backbones. In Proceedings of INFOCOM '06, 2006.

16. A. Adya, P. Bahl, J. Padhye, A. Wolman, and L. Zhou. A multi-radio unification protocol for IEEE 802.11 wireless networks. In Proceedings of Broadnets '04, pp. 344 354,2004

17. R. Draves, J. Padhye, and B. Zill. Comparison of routing metrics for static multi-hop wireless networks. In Proceedings of SIGCOMM '04, pp. 133-144, New York, NY, USA, 2004

18. D. De Couto, D. Aguayo, J. Bicket, and R. Morris. A high-throughput path metric for multi-hop wireless routing. In Proceedings of MOBICOM '03, San Diego, CA, USA, 2003.

19. C. E. Koksal and H. Balakrishnan. Quality aware routing in time-varying wireless networks. in IEEE Journal on Selected Areas of Communication Special Issue on Multi-Hop Wireless Mesh Networks. vol. 24, no. 11, pp. 1984-1994, November 2006.

20. T. Lopatic. OLSRD link quality extensions. Internet page: http://www.olsr.org/docs/READMELink- Quality.html, December 2004. 
21. R. Draves, J. Padhye, and B. Zill. Routing in multi-radio, multi-hop wireless mesh networks. In Proceedings of MOBICOM '04, New York, NY, USA, pp. 114-128, 2004.

22. B. Awerbuch, D. Holmer, and R. Rubens. The Medium Time Metric: High throughput route selection in multi-rate ad hoc wireless networks. Springer Mobile Networks and Applications, vol. 11, no. 2, pp. 253-266, April 2006.

23. V. Bahl, A crash course in mesh networking. ACM SIGCOMM 2006 tutorial, Pisa, Italy, September 2006

24. Y. Yang, J. Wang, and R. Kravets, Designing routing metrics for mesh networks, Proc. WiMesh, 2005.

25. P. Kyasanur and N.H. Vaidya. Routing and link-layer protocols for multi-channel multi-interface ad hoc wireless networks. SIGMOBILE Mob. Comput. Commun. Rev., vol. 10, no. 1, pp. 31-43, January 2006.

26. C. E. Koksal and H. Balakrishnan. Quality aware routing in time-varying wireless networks. in IEEE Journal on Selected Areas of Communication Special Issue on Multi-Hop Wireless Mesh Networks. vol. 24, no. 11, pp. 1984-1994, November 2006.

27. K. Paul, S. Bandyipadhyay, A. Mukherjee, and D. Saha. Communication-aware mobile hosts in ad-hoc wireless network. IEEE ICPWC '99. Jaipur, India, February 1999.

28. A. Agarwal, A. Ajuja, J.P. singh, and R. Shorey, Route-lifetime based routing (RABR) protocol for mobile ad-hoc networks. IEEE ICC 2000, New Orleans, LA, United States, June 2000.

29. A. Mcdonald and T. Znati. A path availability model for wireless ad-hoc networks. In Proceedings of the IEEE Wireless Communications and Networking Conference, 1998.

30. M. Gerharz, C. de Waal, M. Frank, and P. Martini. Link stability in mobile wireless ad hoc networks. In Proceedings of the 27th Annual IEEE Conference on Local Computer Networks (LCN'02), pp. 30-39, Tampa, FL, USA, November 2002.

31. S. Jiang, D. He, and J. Rao. A prediction-based link availability estimation for mobile ad hoc networks. Proceedings of INFOCOM ‘01, pp. 1745-52, 2001.

32. K. Scott and N. Bambos. Routing and channel assignment for low power transmission in PCS. In ICUPC '96, vol. 2, pp. 498-502, 1996

33. J-P. Sheu, C-T. Hu, and C-M. Chao. The Handbook of Ad Hoc Wireless Networks, Chapter Energy-Conserving Grid Routing Protocol in Mobile Ad Hoc Networks. RCR Press LLC, 2003.

34. N. Gupta and S.R. Das. Energy-aware on-demand routing for mobile ad hoc networks In Proceedings of 4th International Workshop on Distributed Computing, Mobile and Wireless Computing, volume 2571, pp. 164-173, January 2002.

35. S. Singh, M. Woo, and C. Raghavendra. Power-aware routing in mobile ad hoc networks. In The Fourth Annual ACM/IEEE International Conference on Mobile Computing and Networking, pp. 181-190, 1998.

36. C.-K. Toh. Maximum battery life routing to support ubiquitous mobile computing in wireless ad hoc networks. IEEE Communications Magazine, pages 2-11, June 2001.

37. D. Kim, J. Garcia-Luna-Aceves, K. Obraczka, J. Cano, and P. Manzoni. Performance analysis of power-aware route selection protocols in mobile ad hoc networks. Proc. IEEE Networks 2002, Atlanta, GA, USA, August 2002

38. J. Chang and L. Tassiulas. Maximum lifetime routing in wireless sensor networks. In Proceedings of $37^{\text {th }}$ Annual Allerton Conference on Communication, Control, and Computing, Monticello, IL, September 1999

39. J. Chang and L. Tassiulas. Energy conserving routing in wireless ad-hoc networks. Proceedings of INFOCOM '00, Tel Aviv, Israel, March 2000. 
40. A. Michail and A. Ephremides. Energy-efficient routing for connection-oriented traffic in wireless ad-hoc networks. Mobile Networks and Applications, vol. 8, no. 5, pp. 517-533, October 2003.

41. IEEE, "Draft amendment: ESS mesh networking," IEEE P802.11s Draft 1.00, November 2006

42. IEEE, “Draft amendment", IEEE P802.11k/D9.0, Sep 07. 